\title{
Efficacy of imidacloprid + moxidectin and selamectin topical solutions against the KS1 Ctenocephalides felis flea strain infesting cats
}

\author{
Michael W Dryden ${ }^{1 *}$, Patricia A Payne ${ }^{1}$, Vicki Smith ${ }^{1}$ and Joe Hostetler ${ }^{2}$
}

\begin{abstract}
Background: Two studies were conducted to evaluate and compare the efficacy of imidacloprid + moxidectin and selamectin topical solutions against the KS1 flea strain infesting cats. In both studies the treatment groups were comprised of non-treated controls, $6 \% \mathrm{w} / \mathrm{V}$ selamectin (Revolution ${ }^{\circledR}$; Pfizer Animal Health) topical solution and 10\% $\mathrm{w} / \mathrm{v}$ imidacloprid $+1 \% \mathrm{w} / \mathrm{v}$ moxidectin (Advantage Multi ${ }^{\circledR}$ for Cats, Bayer Animal Health) topical solution. All cats were infested with 100 fleas on Days $-2,7,14,21$, and 28 . The difference in the studies was that in study \#1 efficacy evaluations were conducted at 24 and 48 hours post-treatment or post-infestation, and in study \#2 evaluations were conducted at 12 and 24 hours.

Results: In study \#1 imidacloprid + moxidectin and the selamectin formulation provided $99.8 \%$ and $99.0 \%$ efficacy at 24 hours post-treatment. On day 28, the 24 hour efficacy of the selamectin formulation dropped to $87.1 \%$, whereas the imidacloprid + moxidectin formulation provided $98.9 \%$ efficacy. At the 48 hour assessments following the 28 day infestations, efficacy of the imidacloprid + moxidectin and selamectin formulations was $96.8 \%$ and 98.3\% respectively. In study \# 2 the efficacy of the imidacloprid + moxidectin and selamectin formulations 12 hours after treatment was $100 \%$ and $69.4 \%$, respectively. On day 28 , efficacy of the imidacloprid + moxidectin and selamectin formulations 12 hours after infestation was $90.2 \%$ and $57.3 \%$, respectively. In study \#2 both formulations provided high levels of efficacy at the 24 hour post-infestation assessments, with selamectin and imidacloprid + moxidectin providing $95.3 \%$ and $97.5 \%$ efficacy, following infestations on day 28.

Conclusions: At the 24 and 48 hour residual efficacy assessments, the imidacloprid + moxidectin and selamectin formulations were similarly highly efficacious. However, the imidacloprid + moxidectin formulation provided a significantly higher rate of flea kill against the KS1 flea strain infesting cats at every 12 hour post-infestation residual efficacy assessment. Both formulations should provide excellent flea control for an entire month on cats.
\end{abstract}

\section{Background}

Numerous animals that travel through neighborhoods, parks and yards carry Ctenocephalides felis (cat fleas), including feral cats and wildlife, such as opossums, raccoons, foxes, and coyotes [1]. These flea-infested animals are continuously depositing flea eggs in the outdoor environment. Within a few weeks, eggs deposited in protected areas may develop into adult fleas. If pets come in contact with these areas, they can rapidly acquire fleas. Once on a pet, the fleas will feed and

\footnotetext{
* Correspondence: Dryden@vet.k-state.edu

'Dept. of Diagnostic Medicine/Pathobiology Kansas State University Manhattan KS 66506, USA

Full list of author information is available at the end of the article
}

mate, after which female fleas will begin laying eggs within as little as 24 hours [2]. After a few days, each female flea will produce 40 to 50 eggs per day, with hundreds and potentially thousands of eggs being deposited back in the home [3].

Most pet owners never see the first two or three fleas their pets acquire and pets often go without flea preventive treatment for several days or weeks [4]. During that time, flea eggs are continually deposited in the home, with development of larvae, pupae, and eventually emerging adult fleas. At some point, there are enough fleas emerging within the home and on the pet that the problem becomes noticeable by the owner. But by that time, the home is already infested with hundreds to

\section{Biomed Central}


thousands of immature flea life stages that develop and emerge as adults to continually reinfest the pet $[4,5]$.

When the flea infested pet is presented to a veterinarian, the resident flea population on the pet must be eliminated rapidly, but control of the infestation is ultimately achieved by eliminating the existing immature and mature flea life stages within the premises. As fleas emerge and jump on pets, it is critical to kill them as rapidly as possible. The more rapidly a residual flea product can kill newly acquired fleas, the more effectively it can manage flea allergy dermatitis, the more likely it can decrease the transmission of vector borne diseases, and fewer fleas are observed by pet owners [6]. An added benefit of an effective residual flea product is its contribution to reproductive suppression, attempting to kill newly acquired fleas before they can reach reproductive status [6].

The purpose of the two studies described in this article was to evaluate the initial and residual speed of kill of an imidacloprid + moxidectin topical formulation against the KS1 Ctenocephalides felis, flea strain infesting cats. The imidacloprid + moxidectin formulation was compared against a topical selamectin formulation, which had previously been evaluated against the KS1 cat flea strain. The KS1 cat flea strain has been maintained as a closed colony at Kansas State University since 1990. Previous studies have indicated that the KS1 strain has some level of resistance or reduced susceptibility to carbaryl, chlorpyriphos, fenthion, fipronil, imidacloprid, permethrin, pyrethrins, and spinosad [6-13].

\section{Methods}

The two studies conducted in these investigations had overall similar study designs. The primary difference between the studies was that in study \#1 speed of kill was evaluated at 24 and 48 hours post-treatment or post-infestation. Whereas in study \#2, speed of kill was assessed at 12 and 24 hours post-treatment or post-infestation.

\section{Animals and housing}

Study one (1) included the use of 34 purpose bred Domestic Short Hair cats (17 m:17 f) and study two (2) used $56(28 \mathrm{~m}: 28 \mathrm{f})$ purpose bred DSH cats between 7 and 12 months of age. The cats were housed in standard stainless steel cages. No drugs, baths, shampoos, or pesticides were administered to the cats during the preconditioning phase or during the course of the study, other than what was described in the protocol. All animal care procedures conformed to guidelines established by the Institutional Animal Care and Use Committee at Kansas State University (IACUC \# 2833 \& \#2904).

\section{Animal Selection and Randomization}

On day -7 , cats in each study were infested with 100 cat fleas, C. felis, (KS1 strain) 1 to 5 days post emergence.
On day -5 , flea comb counts were performed to assess the ability of cats to maintain infestations. Cats were combed with a fine-toothed flea comb having 12-13 teeth $/ \mathrm{cm}$. Flea removal was achieved by combing each cat thoroughly for $10 \mathrm{~min}$. If five or more fleas were recovered during this period, the cat was combed for an additional $5 \mathrm{~min}$. If any fleas were recovered during the second combing period, the cats were combed for an additional 5 min.

In study \#1 the 15 male cats and 15 female cats retaining the highest flea levels were retained for the study. In study \#2 the 24 male cats and 24 female cats retaining the highest flea levels were retained for the study. Within each gender the cats were ranked in descending order by flea count. Cats were randomly grouped into replicates of three based on descending flea counts and allocated into one of three treatment groups (Study \# 1-10 cats; $5 \mathrm{~m} / 5$ f: Study \# 2-16 cats 8 $\mathrm{m} / 8 \mathrm{f}$ ). Each group of cats was then randomly divided into two subgroups of 5 cats (Study \#1) or 8 cats (Study \#2) each. While cats were not allocated according to weight, the cats in the treatment groups were similar in size. In study \#1 the cats in the three treatment groups weighed on average $3.27,3.47$ and $3.41 \mathrm{~kg}$. While in study \# 2 the cats in the three treatment groups weighed on average $3.31,3.22$ and $3.24 \mathrm{~kg}$.

\section{Treatments}

In each study, treatment groups were comprised of nontreated controls, $6 \% \mathrm{w} / \mathrm{v}$ selamectin (Revolution ${ }^{\circledR}$; Pfizer Animal Health) topical solution, and $10 \% \mathrm{w} / \mathrm{v}$ imidacloprid $+1.0 \% \mathrm{w} / \mathrm{v}$ moxidectin (Advantage Multi ${ }^{\circledR}$ for Cats, Bayer Animal Health) topical solution. In both studies the products were applied according to label directions.

\section{Efficacy Evaluations}

To evaluate efficacy of the formulations in eliminating an existing flea infestation, all cats were infested with 100 fleas on Day -2 and treatments were applied on Day 0 . In study \#1, efficacy was determined by removing fleas from 5 cats in each treatment group at 24 hours, and 5 cats at 48 hours post-treatment. In study \#2, efficacy was determined by removing fleas from 8 cats in each treatment group at 12 hours, and from 8 cats at 24 hours post-treatment. Residual activity was determined by reinfesting cats with 100 adult fleas on days 7, 14, 21 and 28 post-treatment and then removing live fleas in study \# 1 from 5 cats in each treatment group at 24 hours and 5 cats at 48 hours post-reinfestation. In study \#2 live fleas were removed from 8 cats in each treatment group at 12 hours and 8 cats at 24 hours postreinfestation. Fleas were removed using the previously described flea combing procedure. 


\section{Data analysis}

For both studies, geometric means were calculated following transformation using a logarithmic method (averaging the transformed values, and converting the average using antilog to represent a geometric mean). All counts were modified by adding one (1) to each prior to logarithmic transformation and subtracting one (1) from the antilog value to meaningfully represent the geometric mean for each group.

Log (counts+1) were analyzed with an analysis of covariance (ANCOVA) for each study day where flea counts were measured. The pre-treatment counts were used as a covariate. SAS PROC MIXED from SAS version 9.2 $\left(\mathrm{SAS}^{\circledR}\right.$ Institute, Cary, NC) was used for all analyses. Differences in least square means were determined between all pair-wise combinations of the three treatment groups. The fixed effect of treatment groups was evaluated at the alpha level of 0.05 .

\section{Results}

In study \#1, the imidacloprid + moxidectin and the selamectin topical solutions provided $99.8 \%$ and $99.0 \%$ efficacy within 24 hours of treatment (Table 1 ). When cats were reinfested on day 14 , both formulations provided $\geq$ $98.3 \%$ efficacy within 24 hours post-infestation. On day 28 the efficacy of the selamectin formulation 24 hours post infestation decreased slightly to $87.1 \%$ (Table 1 ) while the imidacloprid + moxidectin topical solution provided $98.9 \%$ control. At the 48 hour assessment following the 28 day infestations both formulations provided $\geq 96.8 \%$ efficacy (Table 1 ).

In study \# 2 the imidacloprid + moxidectin topical solution provided $100 \%$ efficacy within 12 hours posttreatment (Table 2) where as the selamectin topical solution only provided $69.4 \%$ efficacy. Throughout the next 28 days, both formulations provided similar high levels of efficacy at the 24 hour post-infestation assessments, with selamectin and imidacloprid + moxidectin providing reductions of $95.3 \%$ and $97.5 \%$ efficacy at the Day 29 count, respectively (Table 2). However, there were significant differences $(\mathrm{P}<0.05)$ in residual efficacy at the 12 hour post-infestation assessments at every time period, with the imidacloprid + moxidectin formulation providing a more rapid residual speed of kill (Table 2) when compared to the non-treated control group and selamectin treated cats. By day 28 posttreatment, the 12 hour post-infestation efficacy for selamectin was $57.3 \%$, while the imidacloprid + moxidectin formulation provided a $90.2 \%$ reduction in flea populations.

There were no adverse events associated with treatments in either study.

\section{Discussion}

These studies demonstrated that the imidacloprid + moxidectin topical solution provided rapid initial speed of kill against the KS1 flea strain, with $100 \%$ efficacy within 12 hours of treatment. However, the initial speed of kill of selamectin was not quite as rapid. The results for the initial speed of kill of selamectin in this investigation were similar to results from a 2005 laboratory study against the KS1 flea strain; in this current investigation the 12 hour post-treatment efficacy was $69.4 \%$ and the 12 hour post-treatment efficacy in the 2005 study was $59.7 \%$ [10]. It is unknown why selamectin has a slower initial speed of kill, but may be related to the systemic activity of the product and its need to be absorbed across the skin to reach effective blood levels.

In addition the residual efficacy of selamectin in this study against the KS1 flea strain was similar to the residual efficacy observed in the 2005 study [10]. In the current investigation the 12 hour post-infestation residual efficacy assessments efficacy ranged from $99.1 \%$ on day 7 to $57.3 \%$ on day 28 . In the 2005 study, at the same post-treatment time points, the efficacy was $99.4 \%$ and $70.9 \%$. Also in the 2005 study the efficacy observed when fleas were removed 48 hours after the 28 day infestation was $99.0 \%$, [10] while in the current investigation the efficacy at the same time period was $98.3 \%$. Additionally, the 24 hour residual efficacy of selamectin on day 28 in the 2005 study was $90.1 \%$, while in the current investigation it was $87.1 \%$ in study \#1 and $95.3 \%$ in study \#2. Even though these studies were conducted over 5 years apart, the efficacy assessments for selamectin against the KS1 fleas strain were remarkably similar.

Also of interest is the difference in residual efficacy between the imidacloprid + moxidectin topical solution in this study and the $9.1 \% \mathrm{w} / \mathrm{w}$ imidacloprid formulation evaluated in the 2005 study. In the previous 2005 speed of kill study against the KS1 flea strain, the imidacloprid topical solution did not provide a more rapid residual speed of kill at 12 hours post-infestation at 7, 14, 21 or 28 days post-treatment than the selamectin formulation [10]. While, in this current investigation imidacloprid + moxidectin topical solution provided a more rapid residual speed of kill at every 12 hour post-infestation assessment. In 2005 the 12 hour post-infestation efficacy at 21 and 28 days for imidacloprid mono topical solution was $65.2 \%$ and $61.6 \%$, respectively [10]. In this current investigation the 12 hour post-infestation efficacy at 21 and 28 days for imidacloprid + moxidectin was $98.5 \%$ and $90.2 \%$, respectively.

While direct statistical comparison between the current and 2005 study cannot be conducted, it appears that the imidacloprid + moxidectin formulation provides 
Table 1 STUDY \#1: Geometric mean flea counts and percent efficacy relative to nontreated controls for cats treated with an $10 \%$ w/v imidacloprid + 1.0\% w/v moxidectin topical solution or a selamectin (6\% w/v) topical spot-on, 24 and 48 hours after treatment or infestation

\begin{tabular}{|c|c|c|c|c|c|c|c|c|c|c|}
\hline & Day 0 & & Day 7 & & Day 14 & & Day 21 & & Day 28 & \\
\hline Treatment $^{1}$ & Mean \# of fleas ${ }^{2,3}$ & $\%$ efficacy $^{4}$ & Mean \# of fleas & $\%$ efficacy & Mean \# of fleas & $\%$ efficacy & Mean \# of fleas & $\%$ efficacy & Mean \# of fleas & $\%$ efficacy \\
\hline \multicolumn{11}{|c|}{24 hours post-treatment or infestation } \\
\hline Controls & $79.2 \mathrm{a}$ & & $67.4 a$ & & $64.1 \mathrm{a}$ & & $68.6 a$ & & $52.6 \mathrm{a}$ & \\
\hline Selamectin & $0.8 \mathrm{~b}$ & 99.0 & $0.0 \mathrm{~b}$ & 100 & $1.1 \mathrm{~b}$ & 98.3 & $6.0 \mathrm{~b}$ & 91.2 & $6.8 \mathrm{~b}$ & 87.1 \\
\hline Imidacloprid-Moxidectin & $0.1 \mathrm{~b}$ & 99.8 & $0.2 \mathrm{~b}$ & 99.6 & $0.0 \mathrm{~b}$ & 100 & $5.4 \mathrm{~b}$ & 92.1 & $0.6 \mathrm{c}$ & 98.9 \\
\hline \multicolumn{11}{|c|}{48 hours post-treatment or infestation } \\
\hline Controls & $72.7 \mathrm{a}$ & & $69.7 a$ & & $53.5 a$ & & $56.8 \mathrm{a}$ & & $52.9 \mathrm{a}$ & \\
\hline Selamectin & $0.0 \mathrm{~b}$ & 100 & $0.0 \mathrm{~b}$ & 100 & $0.0 \mathrm{~b}$ & 100 & $0.5 b$ & 99.1 & $0.9 \mathrm{~b}$ & 98.3 \\
\hline Imidacloprid-Moxidectin & $0.0 \mathrm{~b}$ & 100 & $0.0 \mathrm{~b}$ & 100 & $0.1 \mathrm{~b}$ & 99.7 & $0.6 b$ & 99.0 & $1.7 \mathrm{~b}$ & 96.8 \\
\hline
\end{tabular}

${ }^{1}$ Each of 5 cats in the control group received no treatment. Each of 5 cats in the $10 \% \mathrm{w} / \mathrm{v}$ imidacloprid $+1.0 \% \mathrm{w} / \mathrm{v}$ moxidectin or selamectin $(6 \% \mathrm{w} / \mathrm{v})$ treatment groups were administered the topical spot-ons, according to label directions on Day 0.

${ }^{2}$ Each cat was infested with 100 adult Ctenocephalides felis from the KS1 strain on days -2, 7, 14, 21 and 28.

${ }^{3}$ Geometric mean \# of fleas recovered from cats per treatment group.

${ }^{4} \%$ efficacy $=(($ geometric mean count control-geometric mean count treatment $) /$ geometric mean count treatment $) \times 100$

a, b, c geometric means within a column with unlike letter superscripts are significantly different $(P<0.05)$. 
Table 2 STUDY \#2: Geometric mean flea counts and percent efficacy relative to nontreated controls for cats treated with an $10 \%$ w/v imidacloprid + 1.0\% $\mathbf{w} /$ v moxidectin topical solution or a selamectin $(6 \% \mathrm{w} / \mathrm{v})$ topical spot-on, 12 and 24 hours after treatment or infestation

\begin{tabular}{|c|c|c|c|c|c|c|c|c|c|c|}
\hline & Day 0 & & Day 7 & & Day 14 & & Day 21 & & Day 28 & \\
\hline Treatment $^{1}$ & Mean \# of fleas ${ }^{2,3}$ & $\%$ efficacy ${ }^{4}$ & Mean \# of fleas & $\%$ efficacy & Mean \# of fleas & $\%$ efficacy & Mean \# of fleas & $\%$ efficacy & Mean \# of fleas & $\%$ efficacy \\
\hline \multicolumn{11}{|c|}{12 hours post-treatment or infestation } \\
\hline Controls & $52.2 \mathrm{a}$ & & $62.2 \mathrm{a}$ & & $56.4 a$ & & $47.5 \mathrm{a}$ & & $48.4 a$ & \\
\hline Selamectin & $16.0 \mathrm{~b}$ & 69.4 & $0.6 \mathrm{~b}$ & 99.1 & $5.3 \mathrm{~b}$ & 90.6 & $18.6 a$ & 60.9 & $20.6 a$ & 57.3 \\
\hline Imidacloprid-Moxidectin & $0.0 c$ & 100.0 & $0.0 \mathrm{c}$ & 100.0 & $0.2 \mathrm{c}$ & 99.7 & $0.7 \mathrm{~b}$ & 98.5 & $4.7 \mathrm{~b}$ & 90.2 \\
\hline \multicolumn{11}{|c|}{24 hours post-treatment or infestation } \\
\hline Controls & $45.1 \mathrm{a}$ & & $56.3 a$ & & $52.4 a$ & & $47.1 \mathrm{a}$ & & $49.6 a$ & \\
\hline Selamectin & $1.6 \mathrm{~b}$ & 96.4 & $0.0 \mathrm{~b}$ & 100.0 & $0.1 \mathrm{~b}$ & 99.7 & $0.5 b$ & 99.0 & $2.3 b$ & 95.3 \\
\hline Imidacloprid-Moxidectin & $0.0 c$ & 100.0 & $0.0 \mathrm{~b}$ & 100.0 & $0.1 \mathrm{~b}$ & 99.8 & $0.0 \mathrm{~b}$ & 100.0 & $1.2 \mathrm{~b}$ & 97.5 \\
\hline
\end{tabular}

${ }^{1}$ Each of 8 cats in the control group received no treatment. Each of 8 cats in the $10 \% \mathrm{w} / \mathrm{v}$ imidacloprid $+1.0 \% \mathrm{w} / \mathrm{v}$ moxidectin or selamectin (6\%w/v) treatment groups were administered the topical spot-ons, Each of 8 cats in the control group receive

${ }^{2}$ Each cat was infested with 100 adult Ctenocephalides felis from the KS1 strain on days -2, 7, 14, 21 and 28.

${ }^{3}$ Geometric mean \# of fleas recovered from cats per treatment group.

${ }^{4} \%$ efficacy $=$ ((geometric mean count control -geometric mean count treatment)/geometric mean count treatment) $\times 100$

a, b, c geometric means within a column with unlike letter superscripts are significantly different $(P<0.05)$. 
a more rapid residual speed of kill against the KS1 flea strain than imidacloprid mono topical solution. Whether the more rapid residual speed of kill is from an additive or synergistic effect of the moxidectin is currently unknown.

It has previously been demonstrated that several flea products do not perform well against the KS1 flea strain either due to resistance or innate reduced susceptibility [6-13]. It has been demonstrated that the residual speed of kill of older pyrethroid and organophosphate based flea products is very poor against this flea strain due to resistance $[6,8,11]$. In addition more modern insecticides such as fipronil, imidacloprid, and spinosad also have reduced activity against the KS1 strain $[7,9,10,12,13]$. These newer insecticides were introduced into the U.S. as flea products 6 years (fipronil and imidacloprid) or 17 years (spinosad) after the KS1 strain was colonized. Various studies using other cat flea strains have reported that the 28-30 day residual efficacy of fipronil, imidacloprid, and spinosad flea products should range from approximately $95 \%$ to $100 \%$ [7,14-21]. However, when these formulations were evaluated against the KS1, strain the 28-30 day residual efficacy was markedly reduced $[7,10,12,13]$. While these insecticides have reduced residual activity against the KS1 strain, dinotefuran, metaflumizone and selamectin topical spot-on formulations have demonstrated excellent residual efficacy against this strain $[10,12,13,22]$.

\section{Conclusions}

Based on the current studies reported here, the imidacloprid + moxidectin combination topical formulation was highly effective against the KS1 flea strain, with rapid residual speed of kill, killing $90.2 \%$ of fleas within $12 \mathrm{hrs}$ after infestation 28 days post-treatment, and should provide effective residual flea control on flea infested cats.

\section{Acknowledgements}

These studies were funded in part by grants from Bayer Animal Health, Shawnee, KS We thank biostatistician, Terry Settje for analysis of the data.

\section{Author details}

${ }^{1}$ Dept. of Diagnostic Medicine/Pathobiology Kansas State University Manhattan KS 66506, USA. ${ }^{2}$ Bayer HealthCare, Animal Health PO Box 390 Shawnee Mission, KS 66201, USA.

\section{Authors' contributions}

MWD conceived, designed, supervised the study and drafted the paper. PAP assisted in design of study, data collection and revision of manuscript; VS coordinated and supervised data collection and entry and revision of manuscript; $\mathrm{JH}$ assisted in design of study, monitoring of study and manuscript revision. All authors reviewed and approved the final manuscript.

\section{Competing interests}

MWD has served as a consultant and has been sponsored to lecture by Bayer Animal Health and Pfizer Animal Health, manufacturers of Revolution ${ }^{\oplus}$ and Advantage Multi ${ }^{\oplus}$ for Cats, products that were evaluated in these investigations. $\mathrm{JH}$ is a veterinarian employed by Bayer Animal Health and these studies were funded in part by and publications fees paid by Bayer Animal Health.

Received: 8 June 2011 Accepted: 13 September 2011

Published: 13 September 2011

\section{References}

1. Blagburn BL, Dryden MW: Biology, treatment and control of flea and tick infestations. Vet Clin N Am 2009, 39(6):1173-1200.

2. Dryden M, Rust M: The cat flea-biology, ecology and control. Vet Parasitol 1994, 52:1-19.

3. Dryden M: Host association, on-host longevity and egg production of Ctenocephalides felis. Vet Parasitol 1989, 34:117-122.

4. Dryden MW: How you and your clients can win the flea control battle. Vet Med Supplement 2009, 17-26.

5. Chin A, Lunn P, Dryden M: Persistent flea infestations in dogs and cats controlled with monthly topical applications of fipronil and methoprene. Aust Vet Pract 2005, 35(3):89-96.

6. Dryden MW: Flea and tick control in the $21^{\text {st }}$ century, challenges and opportunities. Vet Dermatol 2009, 20:435-440.

7. Payne PA, Dryden MW, Smith V, Ridley RK: Effect of $0.29 \%$ w/w fipronil spray on adult flea mortality and egg production of three different cat flea, Ctenocephalides felis (Bouché), strains infesting cats. Vet Parasitol 2001, 102(4):331-340.

8. Bossard RL, Dryden MW, Broce AB: Insecticide susceptibilities of cat fleas (Siphonaptera: Pulicidae) from several regions of the United States. J Med Entomol 2002, 39:742-746.

9. Rust MK, Waggoner M, Hinkle NC, Mencke N, Hansen O, Vaughn M, Dryden MW, Payne P, Blagburn B, Jacobs DE, Bach T, Bledsoe D, Hopkins T, Mehlhorn $\mathrm{H}$ : Development of a larval bioassay for susceptibility of cat fleas (Siphonaptera: Pulicidae) to imidacloprid. J Med Entomol 2002, 39:671-674.

10. Dryden MW, Smith V, Payne PA, McTier TL: Comparative speed of kill of selamectin, imidacloprid, and fipronil-(S)-methoprene spot-on formulations against fleas on cats. Vet Therapeutics 2005, 6:228-236.

11. Bass C, Schroeder I, Turberg A, Field L, Williamson MS: Identification of mutations associated with pyrethroid resistance in the para-type sodium channel of the cat flea, Ctenocephalides felis. Insect Biochem Mol Bio 2004, 34:1305-1313.

12. Dryden M, Payne P, Smith V: Efficacy of selamectin and fipronil / (S)methoprene spot-on formulations applied to cats against the adult cat flea, Ctenocephalides felis, flea eggs and adult flea emergence. Vet Therapeutics 2007, 8:255-262.

13. Dryden MW, Payne PA, Smith V, Kobuszewski D: Efficacy of topically applied dinotefuran formulations and orally administered spinosad tablets against the KS1 flea strain infesting dogs. Intern J Appl Research Vet Med 2010, 9(2):123-128.

14. Werner G, Hopkins T, Shmidl JA, Watanabe M, Kriger K: Imidacloprid, a novel compound of the cloronicotinyl group with an outstanding insecticidal activity in the on-animal treatment of pests. Pharm Res 1995, 31:126.

15. Hopkins TJ, Kerwick C, Gyr P, Woodley I: Efficacy of imidacloprid to remove and prevent Ctenocephalides felis infestations on dogs and cats. Comp Cont Ed Pract Vet 1997, 19:11-16.

16. Jacobs DE, Hutchinson MJ, Krieger KJ: Duration of activity of imidacloprid a novel adulticide for flea control against Ctenocephalides felis on cats. Vet Rec 1997, 140:259.

17. Hutchinson MJ, Jacobs DE, Fox MT, Jeannin P, Postal JM: Evaluation of flea control strategies using fipronil on cats in a controlled simulated home environment. Vet Rec 1998, 142:356-357.

18. Ritzhaupt LK, Rowan TG, Jones RL: Evaluation of efficacy of selamectin and fipronil against Ctenocephalides felis in cats. J Am Vet Med Assoc 2000, 217:1666-1668.

19. Young DR, Jeannin PC, Boeckh A: Efficacy of fipronil/(s)-methoprene combination spot-on for cats against shed eggs, emerging and existing adult cat fleas (Ctenocephalides felis, Bouche). Vet Parasitol 1004, 125:397-407.

20. Franc M, Yao KP: Comparison of the activity of selamectin, imidacloprid and fipronil for the treatment of cats infested experimentally with 
Ctenocephalides felis felis and Ctenocephalides felis strongylus. Vet Parasitol 2007, 143:131-133.

21. Snyder DE, Meyer J, Zimmerman AG, Qiao M, Gissendanner SJ, Cruthers LR, Slone RL, Young DR: Preliminary studies on the effectiveness on the novel pulicide, spinosad, for the treatment and control of fleas on cats. Vet Parasitol 2007, 150:345-351.

22. Dryden M, Payne P, Lowe A, Mailen S, Smith V, Rugg D: Efficacy of a topically applied spot-on formulation of a novel insecticide, metaflumizone, applied to cats against a flea strain (KS1) with documented reduced susceptibility to various insecticides. Vet Parasitol 2008, 151(1):74-79

doi:10.1186/1756-3305-4-174

Cite this article as: Dryden et al: Efficacy of imidacloprid + moxidectin and selamectin topical solutions against the KS1 Ctenocephalides felis flea strain infesting cats. Parasites \& Vectors 2011 4:174.

\section{Submit your next manuscript to BioMed Central} and take full advantage of:

- Convenient online submission

- Thorough peer review

- No space constraints or color figure charges

- Immediate publication on acceptance

- Inclusion in PubMed, CAS, Scopus and Google Scholar

- Research which is freely available for redistribution

Submit your manuscript at www.biomedcentral.com/submit 